\title{
Reflets
}

Revue d'intervention sociale et communautaire

\section{L'offre active de services de santé en français en Ontario : une mesure d'équité}

\section{Louise Bouchard, Marielle Beaulieu et Martin Desmeules}

Volume 18, numéro 2, automne 2012

Les inégalités sociales de santé chez les communautés francophones en situation minoritaire (CFSM) au Canada

URI : https://id.erudit.org/iderudit/1013173ar

DOI : https://doi.org/10.7202/1013173ar

Aller au sommaire du numéro

Éditeur(s)

Reflets, Revue d'intervention sociale et communautaire

ISSN

1203-4576 (imprimé)

1712-8498 (numérique)

Découvrir la revue

Citer cet article

Bouchard, L., Beaulieu, M. \& Desmeules, M. (2012). L'offre active de services de santé en français en Ontario : une mesure d'équité. Reflets, 18(2), 38-65.

https://doi.org/10.7202/1013173ar
Résumé de l'article

Cet article fait état d'une recherche sur l'offre active de services en français en Ontario. L'étude avait pour objectifs de recenser les définitions de l'offre active de services en français; d'identifier les pratiques d'offre active (facilitateurs ou obstacles); d'identifier les indicateurs de mesure de l'offre active et de proposer des recommandations permettant d'améliorer l'offre active de services en français. À cette fin, une revue documentaire, une enquête auprès de professionnels oeuvrant dans le domaine de la planification des services de santé en français ainsi qu'un atelier de cartographie conceptuelle ont été entrepris.
Tous droits réservés @ Reflets, Revue d’intervention sociale et communautaire, 2012
Ce document est protégé par la loi sur le droit d’auteur. L'utilisation des services d'Érudit (y compris la reproduction) est assujettie à sa politique d'utilisation que vous pouvez consulter en ligne. 


\title{
L'offre active de services de santé en français en Ontario : une mesure d'équité
}

\author{
Louise Bouchard ${ }^{1}$ \\ Département de sociologie et d'anthropologie et Institut de recherche \\ sur la santé des populations, Université d'Ottawa
}

\section{Marielle Beaulieu}

Consultante

\section{Martin Desmeules}

Institut de recherche sur la santé des populations, Université d'Ottawa Centre d'histoire des régulations sociales, Université du Québec à Montréal

\section{Résumé}

Cet article fait état d'une recherche sur l'offre active de services en français en Ontario. L'étude avait pour objectifs de recenser les définitions de l'offre active de services en français; d'identifier les pratiques d'offre active (facilitateurs ou obstacles); d'identifier les indicateurs de mesure de l'offre active et de proposer des recommandations permettant d'améliorer l'offre active de services en français. À cette fin, une revue documentaire, une enquête auprès de professionnels œuvrant dans le domaine de la planification des services de santé en français ainsi qu'un atelier de cartographie conceptuelle ont été entrepris.

Mots clés : minorité linguistique; services de santé; offre active; Loi 8 


\section{Abstract}

This article reports a research on active offer of French Language Services (FLS) in Ontario. The study objectives were to identify the definitions of active offer of FLS, to identify the practices of active offer (facilitators and / or barriers), to identify indicators to measure the active offer and propose recommendations to improve the active offer of FLS. To this end, a literature review, a survey of professionals in the field of planning health services in French and a workshop concept mapping has been undertaken.

Key words : linguistic minority; health services; active offer; Law 8

\section{Introduction}

«n vertu de la

Loi 8 adoptée en 1986, les francophones sont en droit de recevoir les services gouvernementaux dans leur langue et de communiquer en français avec le gouvernement de l'Ontario. "
L'offre active des services de santé en français est un concept clé au cœur des travaux du Commissariat aux services en français (CSF) de l'Ontario depuis sa création en 2007. Ce thème traverse les nombreux rapports du commissaire qui reconnait les promesses d'une telle approche pour l'amélioration des services de santé aux francophones et, ultimement, pour le respect des cadres législatifs des services publics en français. En vertu de la Loi 8 adoptée en 1986, les francophones sont en droit de recevoir les services gouvernementaux dans leur langue et de communiquer en français avec le gouvernement de l'Ontario. Dans le domaine de la santé, le premier centre médico-social communautaire voit le jour en 1988 à Toronto, suivi de plusieurs autres; un protocole d'entente est signé en 2002 entre le ministère de la Santé et des Soins de longue durée (MSSLD) et le Réseau des services de santé en français de l'Est de l'Ontario; l'affaire Montfort culmine en un investissement majeur pour en faire le seul hôpital d'enseignement francophone de la province; en 2007, la Loi de 2006 sur l'intégration du système de santé local est adoptée et le Conseil consultatif des services de santé en français est mis en œuvre; et enfin, en 2010, le règlement relatif à l'engagement de la collectivité francophone en vertu de 
"Trois éléments de problématique méritent d'être développés pour mettre en lumière l'importance que revêt le concept d'offre active des services en français aujourd'hui : la situation minoritaire; la réforme de la santé et ses nouvelles obligations; et l'équité d'accès aux ressources publiques." la Loi 36 sur les réseaux d'intégration des services de santé est adopté et six entités de planification des services de santé en français sont mises en place (Office des affaires francophones, 2011).

Il faut également souligner l'apport de documents produits par des instances gouvernementales ontariennes, dont l'Office des affaires francophones de l'Ontario, principalement le Practical guide for the active offer of French-language services in the Ontario Government (2008), et d'un document produit par les Services publics de l'Ontario, Framework for Action : A modern public service (2006). Ces deux documents ont contribué à affiner la définition du concept de même qu'à identifier des outils susceptibles d'assurer l'adoption d'une telle approche.

La présente étude sur l'offre active de services de santé en français veut contribuer à cet effort de compréhension et de recherche de solutions pour contrer les inégalités et disparités de santé qui affectent les minorités de langue officielle du pays. Ancrée dans une démarche d'enquête auprès des coordonnateurs des services en français œuvrant au sein des réseaux locaux d'intégration des services de santé (RLISS), cette étude a pour objectifs : 1 . de recenser les définitions de l'offre active de services en français; 2. d'identifier les pratiques d'offre active (facilitateurs ou obstacles); 3. d'identifier les indicateurs de mesure; 4. de proposer des recommandations permettant d'améliorer l'offre active de services en français.

Trois éléments de problématique méritent d'être développés pour mettre en lumière l'importance que revêt le concept d'offre active des services en français aujourd'hui : la situation minoritaire; la réforme de la santé et ses nouvelles obligations; et l'équité d'accès aux ressources publiques.

\section{La situation minoritaire et ses effets sur le développement des communautés}

À l'instar de Roy (2006), nous préconisons l'usage de l'expression "groupes en situation minoritaire " au lieu de "minorités", et ce, pour marquer le fait que ces derniers font l'objet d'une minorisation; un processus par lequel un groupe peut se voir 
"Le concept de minorisation traduit donc le processus par lequel une population est désavantagée sur le plan de son développement. »

\section{"Le MSSLD}

reconnaît les inégalités entourant l'état de santé de la minorité linguistique francophone en Ontario et son accès aux services. " défavorisé sur le plan de l'accès aux ressources. De plus, comme Roy le souligne (p. 22), l'expression " groupes en situation minoritaire » « a l'avantage de ne plus placer le groupe en position d'infériorité par rapport aux autres groupes et met en évidence le fait que c'est la situation qui crée problème et non le groupe en tant que tel".

Les effets de cette minorisation, à la fois historique, politique et culturelle, ont été bien documentés dans les domaines du droit, de l'éducation et plus récemment dans celui de la santé. Les pratiques d'assimilation à la majorité anglophone ont freiné le déploiement d'institutions fondamentales pour le développement des communautés francophones et ont forcé l'adoption de l'anglais pour l'accession aux services publics. Un autre effet d'importance a été la concentration de la population minoritaire dans des contextes d'éloignement et de plus grande précarité économique, avec pour conséquence une population plus âgée, moins éduquée et plus pauvre. Le concept de minorisation traduit donc le processus par lequel une population est désavantagée sur le plan de son développement. Dans le domaine de la santé, la recherche met nettement en lumière le rôle des déterminants sociaux, principalement celui de la position sociale, sur la mauvaise santé, la morbidité et la mortalité (Commission des déterminants sociaux de la santé - CDSS, 2008). Suivant ce cadre d'analyse, nos travaux antérieurs ont montré que les disparités de santé entre anglophones et francophones en Ontario sont avérées (Bouchard et Desmeules, 2011). Le MSSLD reconnaît les inégalités entourant l'état de santé de la minorité linguistique francophone en Ontario et son accès aux services. Il est déterminé à mettre en œuvre des mesures assurant une équité en santé pour les francophones de la province.

Le système de santé devrait être guidé par un engagement à l'égard de l'équité et [par] un respect de la diversité des collectivités lorsqu'il dessert la population de l'Ontario et respect [er] les exigences de la Loi sur les services en français lorsqu'il dessert les collectivités francophones. (MSSLD, 2011) 
Malgré ce que prévoit la Loi sur les langues officielles, l'égalité de traitement demeure dans les pratiques un défi toujours à relever. Une approche d'offre active systématique de services en français, c'est-à-dire une offre qui précède la demande, pourrait s'avérer une mesure d'équité d'accès aux services adressés à la population francophone.

\section{La réforme de la santé de l'Ontario et la création des RLISS}

«...la Loi 36 sur les réseaux d'intégration des services de santé (2006)... Cette réforme de santé offre pour la francophonie minoritaire une opportunité de faire valoir ses droits historiques et l'importance de la langue comme mesure essentielle de la qualité des services. De plus, elle fait valoir l'importance de la participation citoyenne à la définition des besoins et à leur gestion."
Les constats de l'accroissement des inégalités de santé, de la progression constante des coûts d'une médecine hautement technologique et du rendement même du système ont insufflé un vent de restructuration des systèmes de santé selon une vision qui réponde adéquatement aux besoins des communautés. Alors que les systèmes de santé se sont structurés à leur origine autour de l'hôpital et des différentes spécialisations médicales affairées chacune à prendre en charge la maladie, les réformes tendent à renverser ce processus pour mieux coordonner les services et remettre le patient, la personne, au centre du système (Organisation mondiale de la santé - OMS, 2008). L'amélioration de la qualité des soins ainsi que l'utilisation judicieuse des ressources constituent les éléments-clés du virage des soins primaires et communautaires comme "pivot et élément-clé du système de santé » (OMS, 1978; Commission sur l'avenir des soins de santé au Canada, 2002, p. 130).

À l'instar des autres réformes de santé au Canada, la Loi 36 sur les réseaux d'intégration des services de santé (2006) emprunte aussi cette voie en créant quatorze RLISS destinés à mieux répondre aux besoins de santé des communautés.

Cette réforme de santé offre pour la francophonie minoritaire une opportunité de faire valoir ses droits historiques et l'importance de la langue comme mesure essentielle de la qualité des services. De plus, elle fait valoir l'importance de la participation citoyenne à la définition des besoins et à leur gestion. L'offre active de services en français s'ancre dans la philosophie de la réforme qui consiste à rapprocher les services de la population pour mieux répondre aux besoins des personnes, des familles et des communautés. De 
plus, la réforme de santé met l'accent sur le caractère proactif de l'offre de services plutôt que sur la demande, incluant la prestation effective de services.

Dans ce contexte, l'esprit de la réforme de santé contredit aujourd'hui la politique antérieure de désignation de services en français "là où le nombre le permet ", et ce, en raison de la définition restreinte de cette dernière (en termes de masse populationnelle critique) et de son processus (l'organisation doit faire sa demande). Cette politique de désignation comporte en effet des limites à l'accès véritable aux services de santé pour les francophones. De fait, une telle approche va à l'encontre même des principes d'équité, d'accessibilité et d'égalité soutenus par la Loi sur les services en français : pour une égalité de statut comme de traitement, l'offre partielle et passive de services en français, plus axée sur la demande, n'est définitivement pas appropriée.

\section{L'équité en santé : égalité de statut, égalité de traitement}

Selon la définition devenue classique proposée par M.Whitehead (1991), l'« équité en santé " signifie l'impératif de corriger les variations de santé qui sont non seulement inutiles et évitables, mais de plus considérées inéquitables ou injustes. L'équité dans toutes les politiques apparaît dans les sociétés démocratiques comme la solution pouvant se porter garante de l'épanouissement et de la pleine réalisation des individus autant que des peuples. Atteindre l'équité en santé est donc fondamentalement un processus politique et un engagement envers la justice sociale sous-jacente à l'ensemble des politiques (Starfield, 2006).

"L'équité en santé signifie que les mêmes services, de même qualité et dans les mêmes conditions d'accès, soient disponibles à tous, quelle que soit leur situation. "
L'équité en santé signifie que les mêmes services, de même qualité et dans les mêmes conditions d'accès, soient disponibles à tous, quelle que soit leur situation. Elle constitue un devoir moral de réduire, voire d'éliminer, les inégalités sociales en santé. La situation minoritaire, qu' elle soit linguistique ou culturelle, recèle comme nous l'avons mentionné des éléments de vulnérabilité auxquels le groupe majoritaire n'est pas confronté.

C'est pourquoi la Commission sur l'avenir des soins de santé au Canada (2002) a élargi la définition d'" accessibilité aux 
"...l'offre active de services en français devient une mesure de redressement des inégalités en proposant un moyen concret de rapprocher les services de santé des besoins de la population francophone. L'offre active de services en français représente une mesure d'équité propre à assurer le respect de la loi : pour qu'à l'égalité de statut corresponde une égalité de traitement." services " traditionnellement considérée du point de vue de la durée de l'attente ou de la distance, pour y inclure l'influence de facteurs sociaux et culturels tels que la langue, le sexe, le niveau d'instruction et l'état de santé.Ainsi, la Commission propose dans son rapport (p.171) que Santé Canada soutienne les organisations et les établissements de soins ainsi que les gouvernements provinciaux pour leur permettre de surmonter "l'obstacle linguistique à l'accès aux soins ".

De son côté, en promulguant sa Loi sur l'intégration du système de santé local, le gouvernement de l'Ontario adopte une approche d'équité en santé et précise dans son préambule «que le système de santé devrait être guidé par un engagement à l'égard de l'équité et un respect de la diversité des collectivités lorsqu'il dessert la population et respecte les exigences de la Loi sur les services en français lorsqu'il dessert les collectivités francophones » (Gouvernement de l'Ontario, 2006).

Dans ce contexte, l'offre active de services en français devient une mesure de redressement des inégalités en proposant un moyen concret de rapprocher les services de santé des besoins de la population francophone. L'offre active de services en français représente une mesure d'équité propre à assurer le respect de la loi : pour qu'à l'égalité de statut corresponde une égalité de traitement.

\section{Méthodologie}

Outre une revue documentaire sur le thème de l'offre active, l'étude s'appuie sur une enquête auprès du premier groupe de professionnels attitrés à la fonction de coordination régionale des services en français (les coordonnateurs des services en français auprès des RLISS actuels) au moyen d'un questionnaire autoadministré et d'un atelier de cartographie conceptuelle, une méthode de recherche participative.

Le questionnaire servait à recueillir les points de vue personnels des coordonnateurs entourant les services en français et leurs enjeux : l'offre et la demande, l'offre active, les fournisseurs et 
" La cartographie conceptuelle... est une méthode de recherche participative permettant de découvrir les représentations que se fait un groupe d'acteurs d'un sujet donné.»

"L'exercice a permis de formuler 117 énoncés, lesquels ont été classés en 9 regroupements - ou grappes... » finalement leurs responsabilités et rôles dans l'offre active de services. Divisé en cinq sections et comprenant une quinzaine de questions, le questionnaire a été envoyé aux coordonnateurs le 15 mars 2011.

La cartographie conceptuelle, pour sa part, est une méthode de recherche participative permettant de découvrir les représentations que se fait un groupe d'acteurs d'un sujet donné. À cette fin, nous avons réuni les coordonnateurs de services en français à un atelier de travail d'une durée d'une journée. Dans un premier temps, un exercice visait à générer un maximum d'idées ou de dimensions pouvant être reliées aux services en français, et ce, à partir d'un énoncé maître qui se formulait ainsi : "Quand je pense à l'offre active de services de santé en français,je pense... » Dans un second temps, tous les énoncés produits ont été remis aux participants qui, individuellement et selon ce qui leur venait à l'esprit, avaient à les regrouper et à leur accorder une étiquette. Les résultats de ce dernier travail ont été traités statistiquement au moyen d'analyses multivariées d'étalonnage multidimensionnel et d'une analyse hiérarchique ascendante qui ont permis de réduire les énoncés et les regroupements individuels en une carte conceptuelle collective. Les participants ont été réunis à nouveau pour déterminer le nom des nouveaux concepts qui en ont résulté.

L'exercice a permis de formuler 117 énoncés, lesquels ont été classés en 9 regroupements — ou grappes - thématiques illustrant la structure conceptuelle du problème de l'offre active des services en français :

1. la responsabilisation vis-à-vis des services en français;

2. l'accessibilité des services en français;

3. l'intégration des services en français;

4. l'identification de la population francophone;

5. la sensibilisation aux services en français;

6. les défis de l'offre et de la demande;

7. l'engagement de la communauté;

8. les ressources humaines;

9. le financement et les incitatifs. 
Le dendrogramme suivant illustre le résultat de cette classification que l'on peut comparer à un arbre constitué du tronc duquel se séparent les branches (les regroupements ou grappes) et les feuilles (les énoncés). Dans cet exemple, le nombre optimal de regroupements (9) se situe statistiquement au-dessus de la ligne.

\section{Dendrogramme de la formation des concepts}

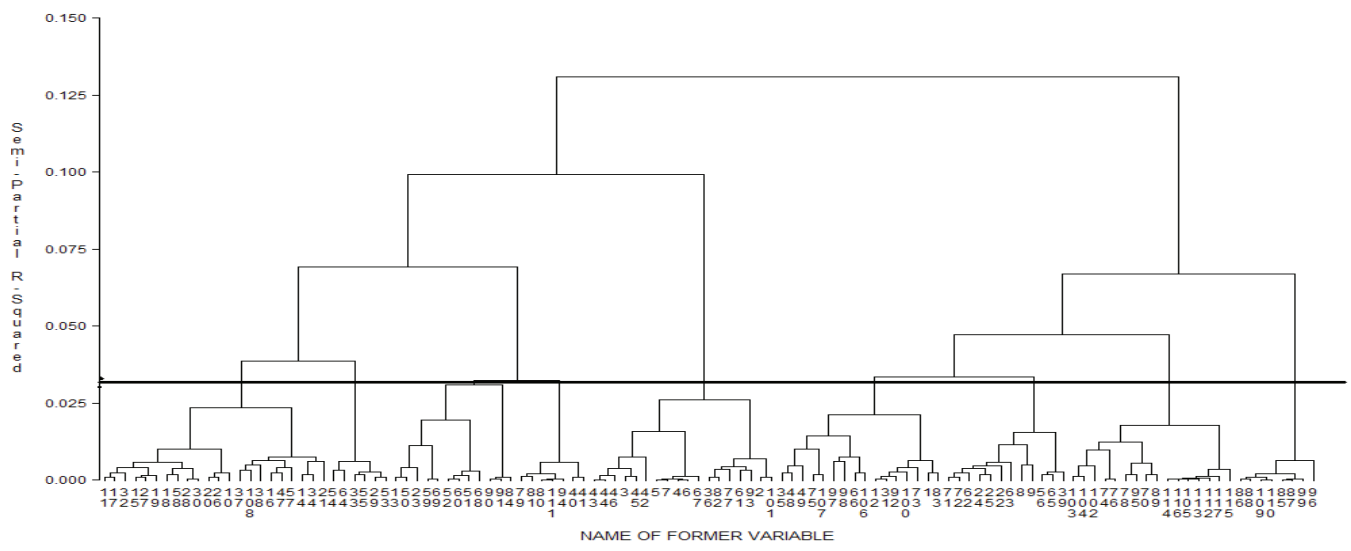

Ces énoncés et regroupements servent de matériaux qualitatifs des représentations sur la problématique de l'offre active. La référence de chacun des énoncés utilisés est indiquée entre parenthèses, d'abord par le numéro de grappe suivi du numéro de l'énoncé (Annexe 1).

\section{Une définition consensuelle et opérationnelle de l'offre active}

"...l'offre active peut être considérée comme une invitation, verbale ou écrite, à s'exprimer dans la langue officielle de son choix."
Au premier abord, l'offre active peut être considérée comme une invitation, verbale ou écrite, à s'exprimer dans la langue officielle de son choix. L'offre de parler dans sa langue officielle de son choix doit précéder la demande de services. Pour qu'il y ait offre active, il faut que l'offre soit visible, audible, accessible (par la parole) et évidente (grappe 2; énoncé 26) et que l'accueil et les services aux francophones soient automatiques, comme un réflexe, et sans délai (grappe 2; énoncé 30). 
Ce premier aspect insiste sur le caractère visible et audible de l'offre qui précède la demande et la suscite. Il rejoint la définition proposée par le CSF :

Essentiellement, le concept de l'offre active est une indication claire aux citoyens que, peu importe où ils se trouvent, ils peuvent recevoir des services en français puisque le nom de l'établissement ainsi que toutes les affiches, pancartes, dépliants, documents, etc., sont soit bilingues, soit offerts visuellement en anglais et en français. De plus, le personnel au comptoir ou au téléphone offre activement, dès la première interaction, un service dans ces deux langues. En somme, il s'agit de créer un environnement qui suscite la demande et anticipe les besoins spécifiques des francophones et de leur communauté. (2010, p. 11)

Ces aspects de visibilité, d'accessibilité et de disponibilité de services offerts dans leur langue aux francophones vivant en situation minoritaire sont intimement liés aux besoins d'outils (pancartes, affiches, dépliants, etc.), de traduction et de professionnels bilingues. Ces aspects sont évidemment importants, mais ils ne fournissent pas une définition complète du concept, notamment en éludant l'aspect de la livraison de services, au-delà de la seule invitation verbale ou écrite. Il apparaît donc opportun d'insister sur les aspects de livraison effective et de qualité des services de santé en milieu linguistiquement minoritaire. L'offre

"...non seulement faut-il offrir activement les services en français, mais il faut s'assurer que leur accessibilité et leur prestation soient de qualité équivalente à ceux offerts en anglais..." active inclut aussi la capacité de livrer la marchandise en français (grappe 2; énoncé 38).

En fait, non seulement faut-il offrir activement les services en français, mais il faut s'assurer que leur accessibilité et leur prestation soient de qualité équivalente à ceux offerts en anglais (grappe 2; énoncé16).

Pour nos répondants, la question de l'accessibilité est une dimension-clé de l'offre active. Elle constitue le deuxième regroupement conceptuel en importance après la dimension de 
la responsabilisation. On y mentionne que les services en français doivent être offerts automatiquement, comme un réflexe (grappe 2; énoncé 30); qu'ils doivent être disponibles dès l'admission du patient et tout au long de son parcours (grappe 2; énoncé 34); ainsi que dans tout le continuum de soins (grappe 2; énoncé 14). On souhaite que les services en français soient accessibles de la même manière et dans les mêmes délais que les services dans la langue de la majorité (grappe 2; énoncé 16). Ainsi, l'offre active de services en français est considérée comme une bonne pratique, la bonne chose à faire (grappe 2; énoncé 57), voire la solution au problème de l'accès (grappe 2; énoncé 37), qui de surcroît met le client au centre de l'intervention (grappe 2; énoncé 17) tel qu'énoncé dans les priorités du MSSLD.

À ce chapitre, la définition proposée dès 2006 par les Services publics de l'Ontario et reprise par l'Office des affaires francophones de l'Ontario (2008, p. 3) complète bien les premiers éléments jusqu'ici identifiés :

The OPS [Ontario Public Services] Framework for Action: A Modern Ontario Public Service, released in 2006, speaks of an 'active offer' as follows: 'High quality modern public services also include an active offer and delivery of French-language services to Ontario's Francophone community. The OPS is effective in fulfilling its responsibilities under the FLS [French Language Services] Act when Francophone members of the public are informed about available services in French, have access to these services and are satisfied with the quality of these services.

"...ils soulignent ainsi les impacts négatifs d'une couverture de services en français incomplète ou de qualité moyenne sur la qualité des soins et des services. "
De l'avis des répondants, certaines pratiques, souvent parce qu'incomplètes, représentent un frein à l'accessibilité et à la qualité des services de santé en français. Pour plusieurs participants, le lien entre qualité de la langue et qualité des services de santé est clair; ils soulignent ainsi les impacts négatifs d'une couverture de services en français incomplète ou de qualité moyenne sur la qualité des soins et des services. On mentionne à ce titre les limites des traducteurs automatisés, tels Google Translate ou Pocket Translate, pour assurer la qualité des services aux francophones. 
En conclusion, les coordonnateurs des services en français des divers RLISS témoignent de manière évidente de leur intérêt à développer l'offre active pour assurer une meilleure accessibilité et une qualité optimale des services de santé aux francophones. Il s'agit dans ce sens de donner aux services en français la place qui leur revient quant à la qualité et à l'équité des soins (grappe 3; énoncé75), mieux encore de les considérer comme tout service public essentiel (grappe 3; énoncé 66).

\section{L'offre active de services en français : une alternative à l'offre « là où le nombre le permet "}

"...une offre de services en français non pas basée sur la demande ou le nombre, mais intégrée à part entière dans le système de santé. ...tous les services de santé doivent être disponibles dans les deux langues officielles...»
Les participants à notre enquête ont bien relevé le problème relié à l'offre de services basée sur la demande et considèrent que dans une situation minoritaire, c'est plutôt le fait d'offrir les services en français qui pourra stimuler la demande (grappe 6; énoncé 65). Ainsi l'offre active est vue comme une alternative qui ne doit plus se justifier par la masse critique de francophones (grappe 6; énoncé 56). Pour eux, une offre véritablement active doit être automatique (grappe 2; énoncé 47) et le fournisseur de services en français doit être proactif (grappe 3; énoncé 1) en les offrant nonobstant la demande. Ils perçoivent par ailleurs qu'il s'agit d'un rêve des communautés francophones (grappe 6; énoncé 9), car les services en français ne sont pas considérés comme une priorité dans le système de santé (grappe 6; énoncé 71).

Conscients que de nombreux organismes se justifient de ne pas offrir les services en français en raison de la faible demande ou de l'absence de demande, les coordonnateurs participants plaident en faveur d'une offre de services en français non pas basée sur la demande ou le nombre, mais intégrée à part entière dans le système de santé. En bref, tous les services de santé doivent être disponibles dans les deux langues officielles (grappe 3; énoncé 48) en raison de l'égalité de leur statut (grappe 3; énoncé 49).

En l'état actuel, les répondants jugent que l'offre de services en français est très faible, voire inexistante dans certaines régions. Quant à l'offre active, elle est perçue comme étant plutôt rare (grappe 6; énoncé 8). Du côté de la demande, les résultats de 
l'enquête montrent que les coordonnateurs des services en français l'évaluent comme étant importante.

\section{Un plan de communication et de sensibilisation aux services en français}

L'offre active de services en français comme nouvelle vision des services publics essentiels et parfaitement intégrés au système de santé exige une stratégie de communication et de sensibilisation tant sur le plan politique, que sur celui des organismes et de la communauté (grappe1; énoncé 95). L'offre de services en français doit être proactive non seulement dans la livraison d'un soin ou d'un service, mais également dans l'ensemble du système de santé : le concept d'" offre active » doit être défini, diffusé et connu pour être concret et effectif.

L'esprit général présidant à l'offre active de services en français est celui qui place le client au centre de l'intervention: " the onus is not on the member of the public to request services in French " (MSSLD, 2008). Il y a donc offre active lorsque l'organisme montre par tous les moyens possibles à la communauté et au client qui se présente à ses portes qu'il peut offrir en français des services de qualité : par

"Une offre active implique que l'organisme offre le service plutôt que d'attendre que le client en fasse la demande et que les intervenants identifient les besoins du client quant à la langue de service et s'assurent que les services sont bel et bien livrés. ” l'affichage, par la documentation disponible et par la présence de ressources humaines aptes à desservir les clients francophones. Une offre active implique que l'organisme offre le service plutôt que d'attendre que le client en fasse la demande et que les intervenants identifient les besoins du client quant à la langue de service et s'assurent que les services sont bel et bien livrés.

Le caractère proactif de l'offre est à ce titre répété par les coordonnateurs : il y a offre active lorsqu'il est évident pour les francophones que les services sont disponibles dans leur langue, notamment par l'affichage et la présence de documentation en français (grappe 2; énoncé 20). De plus, il s'avère nécessaire de faire connaitre aux communautés francophones l'existence des services en français et de promouvoir leur utilisation (grappe 7; énoncé 79).

Mais cet exercice de sensibilisation ne s'arrête pas aux communautés francophones. Il y a effectivement intérêt à ce que la 
"...il importe que le concept d'offre active des services en français fasse partie des politiques des organismes... et soit intégré dans la culture organisationnelle, des conseils d'administration jusqu'aux services directs, et qu'on en reconnaisse la valeur ajoutée..." population ontarienne et, au premier chef, les intervenants en santé comprennent les paramètres de l'offre active de services de santé en français. Il faut que les anglophones sachent que les services en français sont un droit, même pour les francophones qui sont bilingues (grappe 5; énoncé 52). Ce pourrait être également un moyen de contrer de possibles attitudes réfractaires quant aux services en français venant de la majorité anglophone (grappe 6; énoncé 25).

Enfin, il importe que le concept d'offre active des services en français fasse partie des politiques des organismes (grappe 3; énoncé 92) et soit intégré dans la culture organisationnelle, des conseils d'administration jusqu'aux services directs, et qu'on en reconnaisse la valeur ajoutée (grappe 3; énoncé 31). De l'avis des coordonnateurs de services en français, une bonne communication de la disponibilité des services de santé offerts dans cette langue aurait l'avantage de favoriser le sentiment d'inclusion des francophones et de les motiver à s'exprimer en français quand ils y ont recours.

Ce besoin de sensibiliser et d'informer la population francophone à son droit à des services en français a par ailleurs récemment fait l'objet d'une recommandation du CSF à l'endroit de la ministre déléguée aux Affaires francophones : "Que le gouvernement mette en place une stratégie de promotion permanente de l'offre de services gouvernementaux en français auprès de la population francophone partout en Ontario " (CSF, 2011a, p. 55).

Aussi, une campagne de communication à l'adresse des francophones doit être planifiée et conçue en fonction de ses destinataires, bref selon les Directives sur les communications en français adoptées le 13 mai 2010 par le Conseil de gestion du gouvernement et le Conseil du Trésor de l'Ontario (Le Droit, 2009).

Dans Un engagement partagé, rapport annuel 2010-2011 (2011a, p. 14), le commissaire salue cette directive, mais prend toutefois soin de souligner le chemin qu'il reste à faire, notamment pour le MSSLD : 
Grâce aux plaintes reçues, le commissaire continuera de rappeler aux ministères ainsi qu'aux organismes classifiés, le caractère obligatoire de la directive. D'ailleurs, il a encore dans sa mire le ministère de la Santé et des Soins de longue durée, qui pèche sur deux plans : la nonéquivalence des pages anglaises et françaises de son site Web et l'unilinguisme anglais du site Web de plusieurs de ses RLISS.

Ces arguments mettent en évidence le rôle qui incombe au MSSLD en matière de communication en français : il doit faire figure d'exemple en assurant une communication en français de qualité et en rappelant le caractère obligatoire de la directive aux organismes et fournisseurs de services de santé.

\section{L'imputabilité et la responsabilisation du MSSLD}

Au-delà des cadres juridiques et des louables intentions, force est de constater que l'offre active de services de santé en français est non seulement peu connue, mais également fort peu effective à l'heure actuelle en Ontario. À plus d'une occasion, le problème de l'imputabilité du système face aux engagements pris concernant les services en français a été soulevé (grappe 1; énoncé 70).

Cette question de la responsabilisation est centrale dans une approche d'offre active de services de santé en français. Elle constitue en effet la première grappe et le thème le plus important de l'exercice de conceptualisation que nous avons mené auprès des coordonnateurs des services en français des RLISS.

On s'attend à ce que les RLISS, en tant qu'agences de la Couronne, respectent la Loi sur les services en français de manière proactive (grappe 1; énoncé 112); qu'ils soient imputables quant à l'offre et à la livraison réelle de services en français (grappe 1; énoncé 114) et en conséquence, que des mesures de responsabilisation vis-à-vis de la Loi sur les services en français soient établies (grappe 1; énoncé 105); en tant que planificateurs du système local de santé, qu'ils responsabilisent les fournisseurs quant à l'offre et à la livraison de services en français (grappe 1; 
énoncé 113) et qu'ils assurent la surveillance de la prestation et de la qualité desdits services (grappe 1; énoncé 117).

Dans le but de responsabiliser les organismes et fournisseurs de services, le CSF en appelle aussi à une intervention des ministères du gouvernement de l'Ontario, nommément dans le cas qui nous occupe, le MSSLD :

C'est maintenant devenu un thème récurrent pour le commissaire que de parler encore de la nécessité absolue de mettre fin aux échappatoires concernant les services au public offerts par des fournisseurs de services privés pour le compte du gouvernement. La Loi [sur les services en français] est pourtant très claire quant aux obligations des ministères de s'assurer que les services offerts à la population soient offerts évidemment en français. (CSF, 2011a, p. 39)

Dans ce contexte, nous considérons qu'il serait tout à l'avantage du MSSLD de préconiser un abord à la fois exemplaire et conséquent des services de santé en français, à savoir une approche résolument proactive. Dans cet ordre d'idées, nous proposons que le MSSLD fasse acte de leadership en instaurant une véritable filière d'imputabilité face aux engagements en matière de services en français et en donnant les moyens nécessaires aux différentes instances impliquées pour assurer le respect de ces engagements.

On pense aussi que la désignation des organismes offrant des services en français doit être encouragée (grappe 1; énoncé 103) et que toutes les organisations offrant des services en français devraient obtenir leur désignation (grappe 1; énoncé 104). Pour en arriver à responsabiliser le système, et plus particulièrement les fournisseurs, les coordonnateurs ont souligné l'intérêt d'évaluer et de documenter la qualité des services en français, notamment directement à la source, à savoir auprès de la clientèle francophone. Il pourrait s'agir ici de sondages, de questionnaires ou de fiches d'appréciation entourant les services reçus. Comme courroie de transmission entre le MSSLD et les fournisseurs, les RLISS se 
"...les RLISS se doivent d'assurer la mise en ceuvre et le respect des directives et engagements du gouvernement de l'Ontario.... En corollaire à la responsabilisation viennent la question $d u$ financement et des incitatifs et la nécessité que les services en français soient considérés dans le financement de base des services de santé..." doivent d'assurer la mise en œuvre et le respect des directives et engagements du gouvernement de l'Ontario. Pour ce faire, ils doivent être outillés (financement) et pouvoir mettre en œuvre de réelles mesures d'évaluation (qualité, suivi) auprès des fournisseurs, notamment en encourageant ces derniers à obtenir leur désignation. En corollaire à la responsabilisation viennent la question du financement et des incitatifs et la nécessité que les services en français soient considérés dans le financement de base des services de santé (grappe 9; énoncé 99).

Les efforts des vingt dernières années en matière de désignation sont certes louables, mais les résultats démontrent clairement la nécessité de mettre en place des mécanismes de surveillance des services offerts et des outils d'évaluation basés sur des indicateurs de progrès à intervalles réguliers. Pour effectuer un tel travail, les coordonnateurs des services en français des RLISS, en relation avec les entités de planification, s'avèrent des acteurs incontournables.

Considérant la réponse gouvernementale à une recommandation du CSF dans le sens de la responsabilisation des fournisseurs, il nous est permis de croire que le processus de rectification est enclenché :

Le gouvernement de l'Ontario reconnait l'importance de veiller à ce que les ministères respectent la Loi sur les services en français, y compris dans les situations où des services sont offerts par des tiers au nom du gouvernement ou de ses organismes. Il s'engage à examiner le développement d'un règlement qui aborderait cette question importante. (CSF, 2011a, p. 56)

Dans son rapport 2010-2011, le CSF trace une piste de solution intéressante :

[...] le problème de l'offre active de services en français est un problème endémique dans les services gouvernementaux en Ontario et pourrait être résolu par l'adoption d'une directive claire à cet égard du Conseil de gestion 
du Cabinet et par le Conseil du Trésor. (CSF, 2011a, p. 19)

\section{Les fournisseurs de services et de soins de santé en français}

Au plus près de l'offre de services et de soins se retrouvent les fournisseurs et leur personnel. À ce niveau comme aux autres niveaux jusqu'ici identifiés, il existe des freins à l'établissement et au respect d'une offre active de services en français. De fait, il importe ici de brosser le tableau de ces obstacles et d'identifier les pistes susceptibles d'assurer l'avenir de l'offre active de services de santé en français; en bref, d'assurer le respect de la loi.

D'emblée, le niveau d'engagement des fournisseurs de services de santé a été jugé plutôt sévèrement sur la question des services en français par les coordonnateurs. Il y a un manque d'engagement des pourvoyeurs de services envers les services en français (grappe 6; énoncé 22), il faut que le concept d'offre active fasse partie des politiques des organismes (grappe 3; énoncé 92). En ce sens, le MSSLD et les RLISS doivent assurer que leurs fournisseurs de services soient tenus de mettre en place les services en français désirés et qu'ils aient à en rendre compte.

\section{Des outils pour une offre active de services de santé en français}

Le tableau jusqu'ici brossé, surtout attentif aux lacunes du système à l'égard de l'offre active de services de santé en français, ne pourrait être complet sans le riche apport des coordonnateurs en terme d'outils et de perspectives d'avenir. Bien au-delà des constats, quelque peu négatifs, mais toutefois réalistes, il importe d'insister sur les modalités de développement de l'offre active qui émanent des réflexions des coordonnateurs des services en français des RLISS sur la question. Les outils proposés sont nombreux et variés. Ils portent sur la communication, sur la gestion et sur la formation.

\section{Conclusions et recommandations}

Cette première étape de recherche sur l'offre active, ses méthodes et ses outils, démontre qu'il s'agit là d'un concept clé en matière 
"...les coordonnateurs des services en français des RLISS comprennent bien la notion de l'offre active et qu'ils y voient un complément direct à une implantation plus active des services dans cette langue et de la désignation."

"Le concept d'offre active devient ainsi une mesure d'équité et l'outil d'un changement de culture souhaité...» de services de santé en français qui peut contrer les effets de la minorisation et améliorer les services. Les données obtenues tout au long des différentes étapes de recherche indiquent que les coordonnateurs des services en français des RLISS comprennent bien la notion de l'offre active et qu'ils y voient un complément direct à une implantation plus active des services dans cette langue et de la désignation.

De plus, la réforme sur les services de santé de 2006 (Loi 36) met en évidence l'importance des RLISS à l'échelle des communautés pour mieux répondre à leurs besoins et assurer la durabilité du système de santé et de soins. Cette nouvelle philosophie incite à rapprocher les systèmes de santé des populations contrairement à l'approche précédente qui était d'amener les populations vers les systèmes de santé. De surcroît, le MSSLD doit respecter les exigences de la Loi sur les services en français lorsqu'il dessert les collectivités francophones. Pour sa part, le commissaire des services en français évoque l'absolue nécessité d'améliorer la communication en français et de légiférer en la matière. L'entrée en vigueur en juillet 2010 du Règlement sur la prestation des services en français par les tierces parties pour le compte de ministères et organismes gouvernementaux (Loi sur les services en français, 2011) obligera désormais les organismes qui offrent des services au nom de la province à le faire en français. Le concept d'offre active devient ainsi une mesure d'équité et l'outil d'un changement de culture souhaité, faisant en sorte que la prestation de services en français est bien réelle, et ce, dans le continuum des soins.

Ces derniers événements, conjugués avec les fruits de notre recherche, mettent en évidence le rôle du MSSLD en matière de leadership ainsi que celui des RLISS et des fournisseurs de services en ce qui a trait à leur imputabilité en matière de services en français. Sans cadre de responsabilisation engageant ces acteurs de premier plan, il sera difficile, voire impossible, d'assurer l'implantation de l'offre active même si elle contribue à assurer en français des services de santé de qualité. Un important travail de planification des services de santé en français a été amorcé et le gouvernement de l'Ontario est responsable d'assurer des services en français ainsi que de communiquer dans cette langue. L'intérêt 
démontré par le MSSLD pour assurer des services en français de qualité pave la voie vers de plus amples études et documentations sur la question de l'offre active. Les acteurs sur le terrain que sont les équipes des RLISS, les nouvelles entités de planification des services de santé en français, les fournisseurs de services et la population francophone doivent mettre l'épaule à la roue afin d'assurer de meilleurs services en français; cette collaboration émergente requiert une compréhension commune de l'offre active, une documentation plus importante quant aux outils et aux bonnes pratiques ainsi qu'un plan de communication.

Afin de donner suite aux efforts entrepris jusqu'à maintenant pour assurer les droits constitutionnels du français en Ontario, nous émettons les recommandations suivantes :

Recommandation 1 : Pour une définition opérationnelle de l'offre active

Que le MSSLD se dote d'une définition de l'offre active pour la santé en français et que celle-ci prenne en compte les critères suivants : offre proactive sans demande; visibilité, disponibilité et qualité; équité; approche centrée sur le client; accessibilité à l'ensemble des services de santé dans le continuum de soins.

Recommandation 2 : Pour un plan de communication de l'offre active

Que le MSSLD élabore et mette en œuvre, selon les Directives sur les communications en français, un plan de communication visant à faire connaître le droit à des services de santé en français ainsi qu'à sensibiliser et informer la population et les intervenants en santé sur le concept de l'offre active et ses implications.

Recommandation 3 : Pour une imputabilité du système à l'égard des services en français

Que le MSSLD établisse une filière d'imputabilité du système (gouvernement de l'Ontario, MSSLD, RLISS) à l'égard des services en français et de l'offre active comprenant des mesures d'évaluation de la qualité des services offerts en français, dont celle de la satisfaction des francophones à l'égard des services reçus. 
Recommandation 4 : Pour un engagement des fournisseurs à l'égard des services en français

Que les RLISS prévoient et assurent des ententes avec les fournisseurs de services de santé qui les engagent à développer l'offre active de services en français et à mesurer les progrès et l'atteinte des objectifs.

Recommandation 5 : Pour des outils et des pratiques d'offre active diffusés et partagés.

Que le MSSLD libère des fonds pour assurer la documentation des bonnes pratiques et des outils en matière d'offre active et qu'il en assure l'échange, la diffusion et le partage auprès de tous les intéressés.

\section{Notes}

1 Adresse de correspondance : louise.bouchard@uottawa.ca, Université d'Ottawa. Institut de recherche sur la santé des populations, 1 rue Stewart, pièce 225, Ottawa, ON K1N 6N5, 613-562-5800, poste 1642 .

2 Cet article constitue une version abrégée d'un rapport de recherche préparé pour le Bureau des services de santé en français du ministère de la Santé et des Soins de longue durée de l'Ontario : Louise Bouchard, Marielle Beaulieu, Martin Desmeules (2011). L'offre active de services de santé en français en Ontario : une mesure d'équité, www.rrasfo.ca

\section{Bibliographie}

BOUCHARD, Louise, et Martin DESMEULES (2011). Minorités de langue officielle du Canada : Égales devant la santé?, Montréal, Presses de l'Université du Québec, 118 p.

COMMISSARIAT AUX SERVICES EN FRANÇAIS (2011a). Rapport 2010-2011 : Un engagement partagé, réf. du 22 juillet 2012, http://www.csf.gouv.on.ca/files/files/CSF-rapportannuel-2010-2011.

COMMISSARIAT AUX SERVICES EN FRANÇAIS (2011b). Rapport d'enquête - Dépliant unilingue anglais sur la grippe H1N1: Des communications en voie de guérison, réf. du 31 mai 2011, http:// www.csf.gouv.on.ca.

COMMISSARIAT AUX SERVICES EN FRANÇAIS (2010). Rapport 2009-2010 : L'accès aux solutions, réf. du 11 janvier 2011, http://www.flsc.gov.on.ca/files/files/FLSC-AnnualReport2010Web-21mai.pdf.

COMMISSARIAT AUX SERVICES EN FRANÇAIS (2009a). Rapport spécial sur la planification des services de santé en français en Ontario, réf. du 11 janvier 2011, http://www.csf.gouv.on.ca/ files/Rapport.pdf. 
COMMISSARIAT AUX SERVICES EN FRANÇAIS (2009b). Rapport 2008-2009 : Une voix, des changements, réf. du 11 janvier 2011, http://www.flsc.gov.on.ca/files/FR_csf_rapport_ annuel_08_09.pdf.

COMMISSION DES DÉTERMINANTS SOCIAUX DE LA SANTÉ (CDSS) (2008). Combler le fossé en une génération : instaurer l'équité en santé en agissant sur les déterminants sociaux de la santé, réf. du 24 février 2011, Genève, Organisation mondiale de la santé (OMS), http://www.whqlibdoc. who.int/publications/2009/9789242563702_fre.pdf.

COMMISSION SUR L'AVENIR DES SOINS DE SANTÉ AU CANADA (2002). Guidé par nos valeurs : l'avenir des soins de santé au Canada, Roy J. Romanow, commissaire, Rapport final, Novembre 2002, réf. du 20 juillet 2012, http://publications.gc.ca/collections/Collection/CP32-85-2002F.pdf.

GOUVERNEMENT DE L'ONTARIO (2006). «Loi de 2006 sur l'intégration du système de santé local. Préambule », Service Ontario, Lois-en-ligne, réf. du 24 mai 2011, http://www.e-laws.gov. on.ca/html/statutes/french/elaws_statutes_06104_f.htm\#BK0.

GROUPE DE TRAVAIL SUR LES SERVICES DE SANTÉ EN FRANÇAIS (2005). Services de santé pour la communauté franco-ontarienne : feuille de route pour une meilleure accessibilité et une plus grande responsabilisation, Rapport présenté au ministre de la Santé et des Soins de longue durée de l'Ontario.

MINISTÈRE DE LA SANTÉ ET DES SOINS DE LONGUE DURÉE DE L'ONTARIO (2011) Loi sur l'intégration du système de santé local, réf. du 21 mai 2012, /flhs_lhinf.html.

MINISTÈRE DE LA SANTÉ ET DES SOINS DE LONGUE DURÉE DE L'ONTARIO (2008). Health services providers. French language services. Accountability report and implementation plan, Document de travail.

OFFICE DES AFFAIRES FRANCOPHONES (2011). L'accent sur l'avenir, réf. du 21 mai 2012, http://www.ofa.gov.on.ca/fr/loi-historique.html

OFFICE DES AFFAIRES FRANCOPHONES (2008). Practical guide for the active offer of Frenchlanguage services in the Ontario Government, Gouvernement de l'Ontario.

ORGANISATION MONDIALE DE LA SANTÉ (OMS) (2008). Les soins de santé primaires : maintenant plus que jamais, Rapport sur la santé dans le monde, Genève, OMS.

ORGANISATION MONDIALE DE LA SANTÉ (OMS) (1978). Conférence internationale sur les soins de santé primaires, tenue à Alma-Ata, 6-12 décembre 1978, Genève, OMS.

ROY, Ingride (2006). Vers un droit de participation des minorités à la vie de l'État? Montréal, Wilson \& Lafleur, $406 \mathrm{p}$.

STARFIELD, Barbara (2006). "State of the art in research on equity in health ", Journal of Health Politics, Policy and Law, Vol. 31, No 1, p. 11-32.

WHITEHEAD, Margaret (1991). "The Concepts and principles of equity in health », Health Promotion International, Vol. 6, № 3, p. 217-228. 


\section{ANNEXE 1 : Cartographie de l'offre active}

Les regroupements obtenus à la suite du traitement statistique et validés par les participants sont présentés par ordre d'importance. Les titres (concepts) des grappes ont été formulés par les participants. Les cotes entre parenthèses sont de 1 à $10-10$ étant le plus important. Ses cotes permettent à la fois de voir la moyenne du regroupement et de l'énoncé. Le numéro de l'énoncé sert de référence pour identifier les propos tenus par les participants.

\section{Grappe 1 : Responsabilisation $(8,77)$}

\#112 que les RLISS, en tant qu'agences de la Couronne, respectent la Loi sur les services en français de manière proactive $(9,87)$

\#113 que les RLISS, en tant que planificateurs du système local, responsabilisent les fournisseurs quant aux services en français $(9,47)$

\#114 à l'importance de l'imputabilité des RLISS vis-à-vis des services en français $(9,47)$

\#115 que les RLISS s'assurent que la livraison des services en français soit inscrite dans toutes les initiatives financées par les RLISS $(9,33)$

\#78 que la culture organisationnelle de l'offre active commence au niveau gouvernemental (fédéral et provincial) $(9,33)$

\#117 à l'importance d'assurer une vérification et une évaluation de la qualité des services en français $(9,00)$

\#116 à l'importance d'assurer la responsabilisation des fournisseurs : qualité des services en français et satisfaction des francophones $(8,87)$

\#74 au déséquilibre entre la Loi sur les services en français et le suivi de l'application par le MSSLD $(8,80)$

\#95 à faire connaître le concept d'offre active à tous les niveaux : communauté, gouvernements, organismes, professionnels $(8,80)$

\#105 qu'il doit y avoir des conséquences à la non-application de la Loi sur les services en français $(8,67)$

\#102 qu'on utilise les organismes désignés comme des chefs de file quant aux services en français $(8,33)$

\#103 que la désignation des organismes soit encouragée $(8,27)$

\#76 aux manquements d'application des engagements de la Loi sur les services en français $(8,27)$

\#104 à encourager les organismes francophones à obtenir leur désignation $(8,07)$

\#89 que les RLISS et les organismes comprennent mieux l'organisation d'une offre active de services en français $(8,00)$

\#70 à la nécessité que le système accepte différentes normes pour assurer les services en français $(7,80)$ 


\section{Grappe 2 : Accessibilité aux services en français $(8,73)$}

\#30 que l'accueil et les services aux francophones soient automatiques, comme un réflexe, sans délai $(9,53)$

\#34 que l'offre active soit intégrée dans le processus de suivi du parcours du patient : admission, référence autres services $(9,53)$

\#20 que l'offre de services en français soit visible pour les patients $(9,27)$

\#47 que l'offre active soit automatique $(9,27)$

\#26 que l'offre soit visible, audible, accessible (par la parole) et évidente $(9,20)$

\#14 à l'assurance de recevoir des services, dans un continuum de soins $(9,07)$

\#10 qu'un professionnel s'identifie comme francophone de manière évidente $(9,00)$

\#15 que le patient puisse s'exprimer naturellement en français $(9,00)$

\#21 que les services en français soient accessibles $(9,00)$

\#57 que l'offre active de services en français soit présentée comme la bonne chose à faire, la bonne pratique $(8,93)$

\#37 que l'offre de services en français fasse partie du traitement $(8,80)$

\#11 que le professionnel mette à l'aise le client - patient francophone $(8,73)$

\#16 que les services en français soient disponibles et dans les mêmes délais que les services en anglais $(8,73)$

\#28 que l'offre active soit déclenchée par un geste simple $(8,67)$

\#17 à des services qui mettent le client au centre de l'intervention $(8,53)$

\#27 que l'ambiance mette le patient à l'aise de s'exprimer en français $(8,40)$

\#32 à un personnel sensible aux francophones $(8,40)$

\#38 que l'offre active inclue aussi la capacité de livrer la marchandise en français $(8,40)$

\#108 que l'offre active équivaille au bien-être $(8,20)$

\#18 que les professionnels s'adaptent aux capacités linguistiques du patient $(8,07)$

\#19 que le langage soit simple selon les besoins du patient $(7,73)$

\#53 aux besoins de capacités bilingues adaptées pour les clientèles francophones $(7,67)$

\section{Grappe 3 : Intégration des services en français $(8,43)$}

\#12 à l'offre active comme une culture organisationnelle $(9,67)$

\#32 que la culture organisationnelle, du CA aux services directs, reconnaisse les services en français comme une valeur ajoutée $(9,33)$

\#107 que l'offre active de services en français fasse partie de la qualité des services au même titre que les autres $(9,07)$

\#35 que la langue soit intégrée au système d'information des services de santé $(9,00)$

\#73 que les services en français soient un domaine transversal des services de santé $(8,93)$ 
\#1 que le fournisseur de services en français soit proactif $(8,80)$

\#100 à l'importance de la présence de francophones dans les structures décisionnelles $(8,67)$

\#83 aux politiques des organismes et des fournisseurs qui doivent inclure des postes pour les services en français et leur pérennité $(8,60)$

\#92 que le concept d'offre active fasse partie des politiques des organismes $(8,60)$

\#66 que les services en français soient considérés comme un service public essentiel, comme le service d'électricité $(8,53)$

\#75 qu'il faille donner aux services en français la place qui leur revient quant à la qualité et à l'équité des soins $(8,47)$

\#106 à la nécessité qu'il y ait un protocole pour les anglophones face à un client francophone pour assurer ses services en français $(8,27)$

\#48 à des services de santé disponibles dans les deux langues officielles $(8,20)$

\#49 que le français et l'anglais soient considérés égaux, comme une évidence $(8,20)$

\#98 qu'il faille réfléchir aux raisons de la méconnaissance du concept $(6,87)$

\#97 que l'offre active ne soit pas un concept nouveau $(5,60)$

\section{Grappe 4 : Identification des francophones $(7,78)$}

\#53 que l'identification du francophone soit importante pour l'offre active $(9,20)$

\#29 qu'un organisme reconnaisse sa clientèle francophone $(8,80)$

\#33 à un personnel bien formé pour bien identifier la clientèle francophone dans un continuum de soins $(8,73)$

\#55 à l'importance de poser les bonnes questions pour identifier les francophones $(8,13)$

\#54 aux dangers de la question : préférez-vous recevoir vos services de santé en français ou en anglais? $(6,20)$

\#64 que le français ne soit pas toujours la seconde langue parlée $(5,60)$

\section{Grappe 5 : Sensibilisation $(7,71)$}

\#90 que les exemples d'une bonne offre active soient diffusés et promus $(8,87)$

\#13 qu'il y ait des enjeux importants à ne pas desservir les services en français $(8,73)$

\#91 à documenter les modèles de bonne pratique, les diffuser et les partager $(8,73)$

\#50 que le manque de services en français de qualité ait d'importantes conséquences négatives $(8,67)$

\#52 à l'éducation des anglophones pour qu'ils sachent que les services en français sont un droit, même pour les francophones bilingues $(8,33)$

\#84 aux meilleures pratiques pour les services en français, à leur mise en valeur et à leur diffusion $(7,53)$ 
\#60 à l'importance de sensibiliser anglophones et francophones à l'existence de la culture francophone $(7,27)$

\#68 à sensibiliser les professionnels francophones à l'importance de s'exprimer en français à la mesure de leurs capacités $(7,27)$

\#69 que l'offre active comprenne également une adaptation culturelle aux coutumes et traditions des familles $(7,27)$

\#51 au fait qu'il faut beaucoup d'éducation des francophones pour qu'ils demandent des services en français $(7,07)$

\#23 aux défis en tant que membre de la minorité linguistique en Ontario $(6,53)$

\#59 à l'offre active comme un moyen de préservation culturelle $(6,27)$

\section{Grappe 6 : Défis : offre versus demande $(7,34)$}

\#65 que dans une situation minoritaire, l'offre mène la demande $(9,27)$

\#56 que l'offre active ne doive pas se justifier par une masse critique de francophones $(9,00)$

\#8 le fait que l'offre active est plutôt rare $(8,07)$

\#71 que les services en français ne sont pas considérés comme une priorité dans l'ensemble du système de santé $(7,73)$

\#72 que les services en français ne sont pas considérés comme un programme prioritaire $(7,47)$

\#22 au manque d'engagement des pourvoyeurs de services pour les services en français $(7,13)$

\#25 aux attitudes réfractaires quant aux services en français par la majorité anglophone $(6,93)$

\#62 que le contexte actuel ne favorise pas la valorisation de l'offre active $(6,93)$

\#63 que la planification et l'intégration des services se bornent à la distribution géographique $(6,93)$

\#9 que l'offre active est davantage un rêve des communautés francophones $(6,80)$

\#24 aux difficultés amenées par les changements d'administration pour les services en français $(6,60)$

\#39 que l'offre active est souvent déterminée par la demande active des francophones $(5,20)$

\section{Grappe 7 : Engagement de la communauté francophone $(7,28)$}

\#80 à l'importance de s'intégrer à la communauté (fournisseurs et professionnels) pour développer des liens de confiance $(7,67)$

\#111 que tous les efforts de rapprochement vers les francophones comptent $(7,53)$

\#81 à promouvoir les services en français auprès des communautés en se faisant connaitre directement des communautés francophones $(7,53)$

\#79 à la nécessité de communiquer l'offre de services en français dans les communautés francophones, avec leurs moyens de communication $(7,47)$

\#94 à l'importance de créer ce sens d'appartenance avec la communauté francophone $(7,27)$ 
\#41 que la relation de confiance est importante pour l'offre active de services en français $(6,93)$

\#40 qu'il faut que le client ait confiance dans les organismes pourvoyeurs de services de santé $(6,53)$

\section{Grappe 8 : Ressources humaines $(7,22)$}

\#93 que l'offre active soit considérée comme une attente et une obligation pour les postes désignés bilingues $(9,20)$

\#2 l'empressement souhaité des fournisseurs des services en français à recruter du personnel bilingue de manière proactive $(8,93)$

\#3 au manque de ressources humaines francophones en santé $(8,53)$

\#36 que l'offre de services en français soit intégrée au travail des professionnels de la santé $(8,47)$

\#82 à la communication et la promotion des services en français qui doivent être incluses dans les descriptions de tâches des professionnels $(8,20)$

\#101 que les ordres professionnels jouent un rôle en identifiant leurs professionnels bilingues $(7,87)$

\#45 à l'absence de coordination dans l'affectation du personnel francophone aux patients francophones $(7,67)$

\#61 à l'affectation de personnel bilingue même aux clients francophones qui demandent leurs services en anglais $(7,40)$

\#77 à la nécessité pour les organismes de fournir des ressources, outils, formation, appui à leur personnel bilingue $(7,33)$

\#4 au manque de formation professionnelle en français $(7,20)$

\#6 au manque de formation postsecondaire en français $(7,13)$

\#44 que le professionnel francophone est assujetti à un fardeau plus lourd s'il s'affiche francophone $(6,87)$

\#43 que le professionnel francophone craint de s'afficher de peur d'alourdir sa tâche $(6,67)$

\#42 que l'organisation n'est pas structurée pour intégrer les besoins des tâches en français $(6,27)$

\#46 que les demandes et exigences (traduction, rédaction, communication) aux francophones dépassent souvent leurs compétences $(6,00)$

\#5 aux lacunes du langage spécialisé (clinique) $(5,47)$

\#67 que certains professionnels francophones ne sont pas à l'aise de s'exprimer en français $(5,47)$

\#7 au manque de reconnaissance de la terminologie spécialisée en français $(5,20)$ 


\section{Grappe 9 : Financement et incitatifs $(6,58)$}

\#96 qu'il faut donner les moyens aux coordonnateurs services en français pour développer l'offre active $(7,20)$

\#99 à la nécessité d'intégrer les services en français au financement de base $(7,07)$

\#85 à la problématique du financement des services en français $(6,73)$

\#109 que si le financement réservé aux francophones était identifié, on pourrait s'assurer qu'il est dépensé à bon escient $(6,67)$

\#86 au financement, comme des incitatifs financiers au développement de l'offre active des services en français $(6,67)$

\#87 que l'enveloppe budgétaire actuelle des RLISS ne consacre pas d'argent pour les services en français $(6,40)$

\#88 que le MSSLD fournisse des moyens financiers aux coordonnateurs services en français / RLISS $(6,27)$

\#110 qu'il faudrait que le financement soit identifié selon les populations $(5,60)$ 